University of Nebraska - Lincoln

DigitalCommons@University of Nebraska - Lincoln

John R. Hardy Papers

Research Papers in Physics and Astronomy

2003

\title{
Molecular Dynamics Simulation of the Order-Disorder Phase Transition in Solid $\mathrm{NaNO}_{2}$
}

\author{
Wei-Guo Yin \\ University of Nebraska at Omaha \\ Chun-Gang Duan \\ University of Nebraska at Omaha, cgduan@clpm.ecnu.edu.cn \\ Wai-Ning Mei \\ University of Nebraska at Omaha, physmei@unomaha.edu \\ Jianjun Liu \\ University of Nebraska at Omaha \\ R. W. Smith \\ University of Nebraska at Omaha, robertsmith@unomaha.edu
}

See next page for additional authors

Follow this and additional works at: https://digitalcommons.unl.edu/physicshardy

Part of the Physics Commons

Yin, Wei-Guo; Duan, Chun-Gang; Mei, Wai-Ning; Liu, Jianjun; Smith, R. W.; and Hardy, John R., "Molecular Dynamics Simulation of the Order-Disorder Phase Transition in Solid $\mathrm{NaNO}_{2}$ " (2003). John R. Hardy

Papers. 67.

https://digitalcommons.unl.edu/physicshardy/67

This Article is brought to you for free and open access by the Research Papers in Physics and Astronomy at DigitalCommons@University of Nebraska - Lincoln. It has been accepted for inclusion in John R. Hardy Papers by an authorized administrator of DigitalCommons@University of Nebraska - Lincoln. 
Authors

Wei-Guo Yin, Chun-Gang Duan, Wai-Ning Mei, Jianjun Liu, R. W. Smith, and John R. Hardy

This article is available at DigitalCommons@University of Nebraska - Lincoln: https://digitalcommons.unl.edu/ 


\title{
Molecular dynamics simulation of the order-disorder phase transition in solid $\mathrm{NaNO}_{2}$
}

\author{
Wei-Guo Yin, ${ }^{1,}{ }^{*}$ Chun-Gang Duan, ${ }^{1}$ W. N. Mei, ${ }^{1}$ Jianjun Liu,,${ }^{1,2}$ R. W. Smith, ${ }^{1}$ and J. R. Hardy ${ }^{2}$ \\ ${ }^{1}$ Department of Physics, University of Nebraska, Omaha, Nebraska 68182, USA \\ ${ }^{2}$ Department of Physics and Center for Electro-Optics, University of Nebraska, Lincoln, Nebraska 68588, USA
}

(Received 30 January 2003; revised manuscript received 23 July 2003; published 17 November 2003)

\begin{abstract}
We present molecular dynamics simulations of solid $\mathrm{NaNO}_{2}$ using pair potentials with the rigid-ion model. The crystal potential surface is calculated by using an a priori method which integrates the $a b$ initio calculations with the Gordon-Kim electron gas theory. This approach is carefully examined by using different population analysis methods and comparing the intermolecular interactions resulting from this approach with those from the $a b$ initio Hartree-Fock calculations. Our numerics show that the ferroelectric-paraelectric phase transition in solid $\mathrm{NaNO}_{2}$ is triggered by rotation of the nitrite ions around the crystallographical $c$ axis, in agreement with recent x-ray experiments [Gohda et al., Phys. Rev. B 63, 14101 (2000)]. The crystal-field effects on the nitrite ion are also addressed. An internal charge-transfer effect is found.
\end{abstract}

DOI: $10.1103 /$ PhysRevB.68.174106

\section{INTRODUCTION}

Sodium nitrite is a ferroelectric at room temperature. It has the orthorhombic structure, space group $C_{2 v}^{20}-\operatorname{Im} 2 m$, with the dipole vector of the $\mathrm{V}$-shaped nitrite anions aligned parallel to the crystallographic $b$ direction, as shown in Fig. 1. The ferroelectric-paraelectric phase transition takes place at about $437 \mathrm{~K}$, where the high-temperature phase is orthorhombic, space group $D_{2 h}^{25}$-Immm, with the dipoles disordered with respect to the $b$ axis. In a narrow temperature range from $435.5 \mathrm{~K}$ to $437 \mathrm{~K}$, there exists an incommensurate antiferroelectric phase. The melting temperature is 550 K. Distinguished from displacive ferroelectrics in which the ferroelectric transition is driven by soft phonon modes, $\mathrm{NaNO}_{2}$ offers a model system for research of the orderdisorder structural phase transition and any associated ferroelectric instability. ${ }^{1-3}$

Extensive experimental work on $\mathrm{NaNO}_{2}$ has been devoted to probing the mechanism of the $\mathrm{NO}_{2}^{-}$polarization reversal that triggers the order-disorder transition. The majority of studies support the $c$-axis rotation model, but there were also results favoring the $a$-axis rotation model. ${ }^{4}$ Recently, refined $\mathrm{x}$-ray studies over a wide temperature range reinforced the $c$-axis rotation model. ${ }^{4,5}$ On the theoretical side, the microscopic model calculations done by Ehrhardt and Michel supported the $c$-axis rotation mechanism, ${ }^{6}$ whereas mixed double rotations around the $a$ axis and the $c$ axis were suggested by Kinase and Takahashi. ${ }^{7}$ It has long been desirable to apply computer molecular dynamics (MD) simulations to $\mathrm{NaNO}_{2}$ in order to achieve unambiguous understanding of the polarization reversal mechanism. Earlier MD simulations with empirical Born-Mayer pair potentials detected the $c$-axis rotation in above-room-temperature $\mathrm{NaNO}_{2} \cdot{ }^{8-10} \mathrm{Un}$ fortunately, the low-temperature structure produced by those simulations was antiferroelectric and apparently disagreed with the experimental observations.

$\mathrm{Lu}$ and Hardy pointed out that the overall phase behavior of $\mathrm{NaNO}_{2}$ could be simulated by using an a priori approach to construct the crystal potential surface (PES). ${ }^{11}$ The LuHardy (LH) approach was originally designed to deal with molecular crystals such as $\mathrm{K}_{2} \mathrm{SeO}_{4}$, where there exists a mix
PACS number(s): 64.60.Cn, 61.43.Bn, 64.70.Pf

of bonding types, that is, the intermolecular interactions are mostly ionic, but the constituent atoms in a molecule $\left(\mathrm{SeO}_{4}^{2-}\right.$ in $\mathrm{K}_{2} \mathrm{SeO}_{4}$ ) bond covalently. In the $\mathrm{LH}$ approach, the intramolecule interactions were treated by applying the $a b$ initio self-consistent field method to the gas-phase molecules, while the intermolecular pair potentials were computed within the Gordon-Kim (GK) electron-gas theory. ${ }^{12}$ The crux of their application of the GK theory is how to partition the ab initio molecular charge density among the constituent atoms. Since there is no unique way to separate the charge density of a highly covalently bonded molecule, $\mathrm{Lu}$ and Hardy suggested equal separation in a spirit similar to the Mulliken population analysis (MPA). By using this atomiclevel method, we could successfully describe the phase transitions in fluoroperovskites ${ }^{13}$ and ionic crystals with polyatomic molecules including $\mathrm{SeO}_{4}^{2-},{ }^{14} \mathrm{ClO}_{4}^{-},{ }^{15} \mathrm{SO}_{4}^{2-},{ }^{16}$ $\mathrm{SiO}_{4}^{4-},{ }^{17}$ and $\mathrm{NO}_{3}^{-} \cdot{ }^{18-20}$ Note that the MPA happens to preserve the (zero) dipole moment of these molecules.

However, several problems appear when we moved on to deal with $\mathrm{NaNO}_{2}$ where the $\mathrm{NO}_{2}^{-}$radical has nonzero dipole moment and stronger chemical bonding. First, it is well known that the MPA, while certainly the most widely employed, is also somewhat arbitrary and the most criticized. ${ }^{21}$ In particular, the MPA overestimates the dipole moment of the free $\mathrm{NO}_{2}^{-}$ion by about $120 \%$. Other difficulties involved the free-ion approximation. Unlike in monatomic ionic crystals, there may exist considerable internal charge-transfer effects in molecular ionic crystals. Electronic band-structure calculations ${ }^{22}$ indicated that within a nitrite entity, the nitrogen atom and two oxygen atoms bond covalently, leading to high charge transferability between these constituent atoms. Therefore, in solid $\mathrm{NaNO}_{2}$ the $\mathrm{NO}_{2}^{-}$group will feel different crystal-field environments as it rotates and responds by redistributing the charge density among its three constituent atoms.

Our goals in this paper are twofold. First, we show that our atomistic level simulation methods involving pair potentials with the rigid-ion model is capable of correctly describing the phase behavior of $\mathrm{NaNO}_{2}$. Second, we systematically examine the LH approach to understand why it works so well in molecular ionic crystal systems by the following 


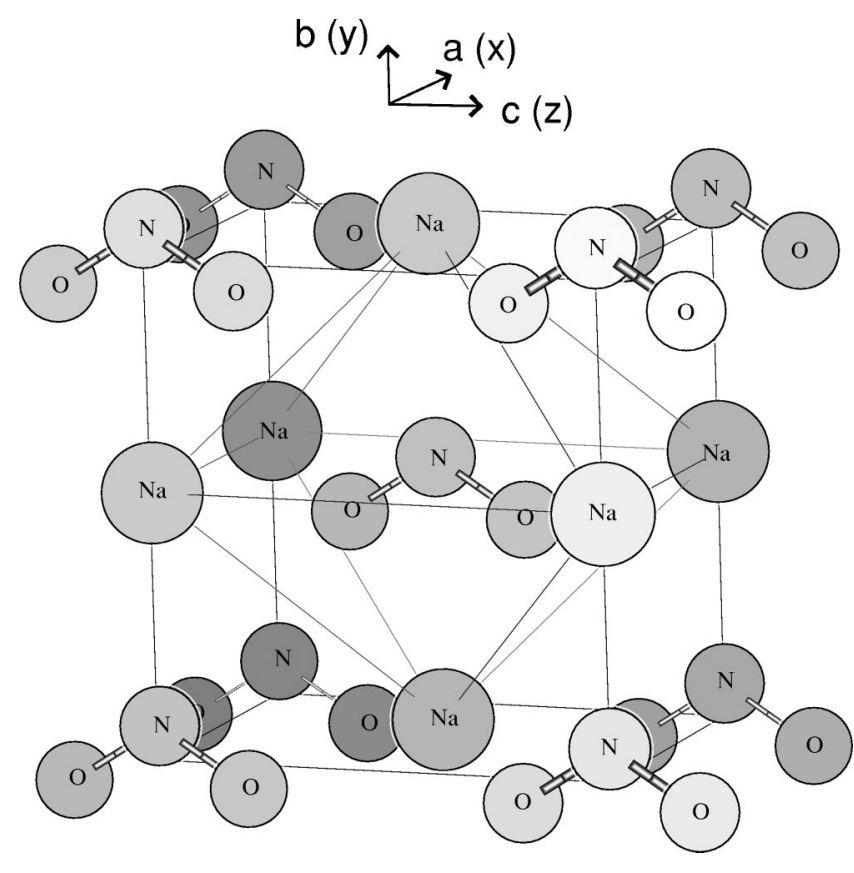

FIG. 1. Crystal structure of $\mathrm{NaNO}_{2}$ in the ferroelectric phase.

steps: (i) we develop another population analysis method that preserves the molecular dipole moment by directly fitting the $a b$ initio charge density of a molecule; (ii) we carry out $a b$ initio Hartree-Fock (HF) calculations of the intermolecular interactions and find that the pair potentials from the rigidion model can correctly reproduce the $a b$ initio results; and (iii) we investigate the crystal-field effects on the $\mathrm{NO}_{2}^{-}$ion by embedding the ion and its first shell of neighbors in a lattice of point charges and find a remarkable internal chargetransfer effect. ${ }^{23}$ Several MD simulations based on these modifications of the LH approach are also performed. The ferroelectric-paraelectric transition triggered by the $c$-axis rotation of the nitrite ions is observed in all versions of the $\mathrm{LH}$ approach. However, the transition temperatures predicted by these simulations are lower than the experimental values. Furthermore, the transition temperatures obtained from the original version are higher than those predicted by modified versions and closer to the experimental values. After careful examination, we notice that in the original LH approach, the $\mathrm{NO}_{2}^{-}$dipole moments were generally enhanced by about $120 \%$. Such enhancement reinforces the ferroelectric state by raising the rotational barriers of $\mathrm{NO}_{2}^{-}$, thus mimicing the anion polarization effect in the mean-field sense. Therefore, we conclude that the anion polarization effect is particularly important for the quantitative study of $\mathrm{NaNO}_{2}$.

This paper is organized as follows. Section II describes the method used to obtain the PES of ionic molecular crystals. Section III analyzes the resulting intermolecular potentials in comparison with those obtained from $a b$ initio calculations. Section IV presents the results of our MD simulations. The crystal-field effects on $\mathrm{NO}_{2}^{-}$are discussed in Sec. V. Concluding remarks are made in Sec. VI.

\section{METHODOLOGY FOR PES CONSTRUCTION}

Our MD simulation technique originates from the GK model for simple ionic crystals such as alkali halides, assum- ing that (molecular) ions in a crystal environment can be described as free ions. ${ }^{24-26}$ Then it was extended to deal with molecular ionic crystals such as $\mathrm{K}_{2} \mathrm{SeO}_{4}$ in which strong intramolecular covalency exists. ${ }^{12,14}$ The main idea is that the molecular ion $\left(\mathrm{SeO}_{4}^{-}\right.$in $\left.\mathrm{K}_{2} \mathrm{SeO}_{4}\right)$ is treated as a single entity, and intramolecular and intermolecular interactions are treated separately: first we perform $a b$ initio quantum chemistry calculations for the whole molecular ion to obtain the optimized structure, the force constants, and the whole electron density $\rho(\mathbf{r})$. The intramolecular interactions are described by force constants within the harmonic approximation. As for the intermolecular interactions, we have to carry out electron population analysis to separate $\rho(\mathbf{r})$ onto each individual atom in the molecular ion, then use the GordonKim electron-gas model to calculate the intermolecular pair potentials. This approach provides a parameter-free description for the crystal potential-energy surfaces, which allow structural relaxation, MD simulation, and lattice dynamics calculations.

In calculating the intermolecular forces, there are three major approximations as discussed in the following.

(1) We assume that the geometries and electronic densities of the separate ions remain unchanged once they have been obtained under given circumstance, such as in the equilibrium state of the gas or crystal phases. This approximation is the fundamental basis for the GK electron-gas theory. Generally speaking, we found that in an ionic crystal there is no strong chemical bond between ions, hence this approximation is reasonable.

(2) When dealing with the intermolecular interaction, we assume that the charge density of a rigid ion can be separated into its atomic constituents.

(3) We assume that the crystal potential energy is composed of the intermolecular and the intramolecular interaction, where the intramolecular interaction is expressed in terms of force fields and the intermolecular interaction is a sum of interatomic pair potentials.

Atomistic level simulations utilizing pair potentials and the rigid-ion model have achieved great success in describing many ionic systems. ${ }^{27}$ We showed that this scheme can correctly describe the phase-transition behaviors of alkali halide fluoperovskites, ${ }^{13}$ and molecular crystals with tetrahedral $^{12,14-17}$ and equilateral triangular ${ }^{18-20}$ radicals. We shall discuss this scheme and its modification in more detail in the remaining part of this section and the following section.

\section{A. Pairwise additive approximation}

In the GK model, we evaluate the interaction between two molecules based on the electron density, ${ }^{28}$ which is approximated as the sum of component densities taken from $\mathrm{HF}$ calculations. That is, if $\rho_{A}$ and $\rho_{B}$ are the component densities, then the total density is $\rho_{A B}=\rho_{A}+\rho_{B}$, and interaction potential is computed as the sum of four terms: Coulombic, kinetic, exchange, and correlation energies which are expressed in terms of the charge densities. 

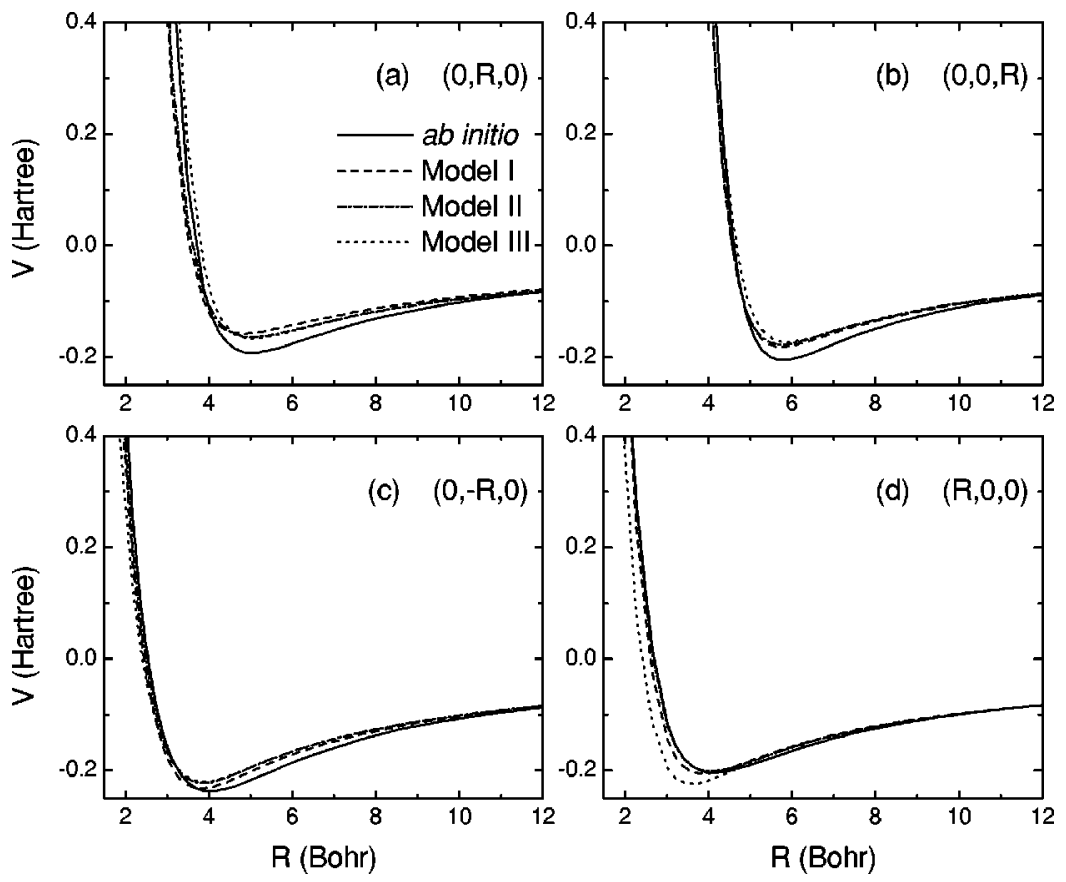

FIG. 2. $\mathrm{NO}_{2}^{-}-\mathrm{Na}^{+}$intermolecular potentialenergy curves as a function of $R$ for various configurations: $(0, R, 0), \quad(R, 0,0), \quad(0,-R, 0)$, and $(0,0, R)$, where $(x, y, z)$ is the location of $\mathrm{Na}^{+}$. Different lines represent the ab initio HF model (solid), model I (dashed), model II (dotted), and model III (dashed and dotted).
Therefore, suppose the $A$ and $B$ molecules are made up of $M$ and $N$ atoms, respectively, then the Coulombic interaction between them is

$$
\begin{aligned}
V_{\mathrm{C}}= & \int d \mathbf{r}_{1} d \mathbf{r}_{2} \frac{\rho_{A}\left(\mathbf{r}_{1}\right) \rho_{B}\left(\mathbf{r}_{2}\right)}{\left|\mathbf{r}_{1}-\mathbf{r}_{2}\right|}-\sum_{i=1}^{M} Z_{A, i} \int d \mathbf{r}_{2} \frac{\rho_{B}\left(\mathbf{r}_{2}\right)}{\left|\mathbf{r}_{2}-\mathbf{R}_{A, i}\right|} \\
& -\sum_{j=1}^{N} Z_{B, j} \int d \mathbf{r}_{1} \frac{\rho_{A}\left(\mathbf{r}_{1}\right)}{\left|\mathbf{r}_{1}-\mathbf{R}_{B, j}\right|}+\sum_{i=1}^{M} \sum_{j=1}^{N} \frac{Z_{A, i} Z_{B, j}}{\left|\mathbf{R}_{A, i}-\mathbf{R}_{B, j}\right|},
\end{aligned}
$$

where $Z_{A, i}, Z_{B, j}, \mathbf{R}_{A, i}$, and $\mathbf{R}_{B, j}$ are the nuclear charges and coordinations of the $i$ th atom in the $A$ molecule and $j$ th atom in the $B$ molecule, respectively. This potential energy can be split into two parts: the long-range part,

$$
V_{\mathrm{C}}^{l}=\sum_{i=1}^{M} \sum_{j=1}^{N} \frac{\left[Z_{A, i}-\int \rho_{A}\left(\mathbf{r}_{1}\right) d \mathbf{r}_{1}\right]\left[Z_{B, j}-\int \rho_{B}\left(\mathbf{r}_{2}\right) d \mathbf{r}_{2}\right]}{\left|\mathbf{R}_{A, i}-\mathbf{R}_{B, j}\right|}
$$

and the short-range part,

$$
V_{\mathrm{C}}^{s}=V_{\mathrm{C}}-V_{\mathrm{C}}^{l}
$$

Equation (2) is essentially the electrostatic interaction energy when the charge densities of the molecules are distributed as point charges on the constituent atoms, which is known as the Madelung potential energy.

The non-Coulombic energy terms are expressed in the uniform electron-gas formula,

$$
V_{i}=\int d \mathbf{r}\left[\rho_{A B}(\mathbf{r}) \epsilon_{i}\left(\rho_{A B}\right)-\rho_{A}(\mathbf{r}) \epsilon_{i}\left(\rho_{A}\right)-\rho_{B}(\mathbf{r}) \epsilon_{i}\left(\rho_{B}\right)\right],
$$

where $\epsilon_{i}(\rho)$ is one of the energy functionals for the kinetic, exchange, and correlation interactions. ${ }^{28}$ Note that Eq. (4) is not composed of pair potentials. In order to obtain the effective pairwise potentials, we approximate Eq. (4) using

$$
\begin{aligned}
V_{i} \simeq & \sum_{m \in A} \sum_{n \in B} \int d \mathbf{r}\left[\rho_{m n}(\mathbf{r}) \epsilon_{i}\left(\rho_{m n}\right)-\rho_{m}(\mathbf{r}) \epsilon_{i}\left(\rho_{m}\right)\right. \\
& \left.-\rho_{n}(\mathbf{r}) \epsilon_{i}\left(\rho_{n}\right)\right],
\end{aligned}
$$

where $\rho_{m n}=\rho_{m}+\rho_{n} . \rho_{m}$ and $\rho_{n}$ are the charge densities of individual atoms in the $A$ and $B$ molecules, respectively, which are obtained by a population analysis as described in the following section.

Even though the non-Coulombic forces as determined by Eq. (4) are not strictly additive, the above approximation appears to be adequate except at very short distances. As pointed out by Waldman and Gordon, ${ }^{29}$ the main reason as to why this approximation is valid is because the Coulombic force, the largest contribution to the potentials, is additive. Based on our calculations, we find additivity of $V_{i}$ holds only to within about $50 \%$; however, the overlap contribution to the electrostatic energy dominates $V_{i}$ and renders additivity to within $10 \%$. One final remark is in order, for the sake of simplifying the two-electron integral in Eq. (1), the charge densities $\rho_{m}$ and $\rho_{n}$ are taken as its spherical average. As a result, the Coulombic interaction is not exactly evaluated. Nevertheless, as we shall show in Figs. 2 and 3, this error is compensated by those due to the pairwise additive approximation.

To summarize this section, we have demonstrated that it is possible to analytically express the intermolecular potentials $V_{\mathrm{C}}^{l}+V_{\mathrm{C}}^{s}+V_{i}$ using Eqs. (2), (3), and (5) once the charge density of each individual atom is obtained by an electronic population analysis.

\section{B. Electronic population analysis and intramolecular interactions}

In this section, we discuss the ways to separate the electron density $\rho(\mathbf{r})$ of a molecule into its atomic constituents. 


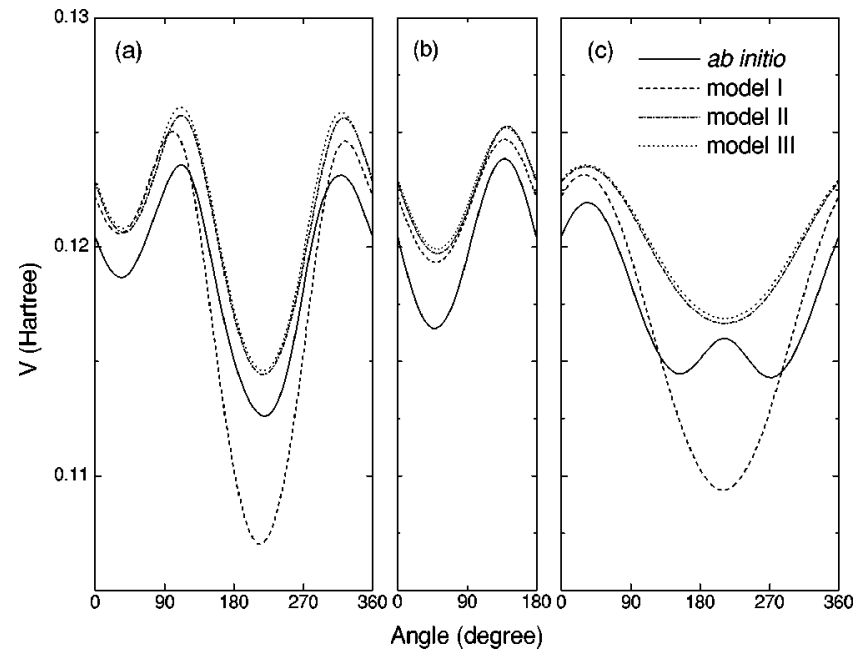

FIG. 3. $\mathrm{NO}_{2}^{-}-\mathrm{NO}_{2}^{-}$intermolecular potential-energy curves as a function of rotation angle. The $\mathrm{NO}_{2}^{-}$molecules are initially in parallel alignment at separation ( $1.82 \AA$, $2.83 \AA$, $2.69 \AA$ ) and then one of them rotates around one of the (a) $x$, (b) $y$, and (c) $z$ axes through its center of mass.

Suppose the molecule consists of $M$ atoms, then the wave function of the molecule $\psi(\mathbf{r})$ can be written as a linear superposition of atomic wave functions $\varphi\left(\mathbf{r}-\mathbf{R}_{i}\right), i$ $=1,2, \ldots, M$, centered at each atom,

$$
\psi(\mathbf{r})=\sum_{i=1}^{M} \varphi\left(\mathbf{r}-\mathbf{R}_{i}\right) .
$$

In turn, the atomic wave functions $\varphi\left(\mathbf{r}-\mathbf{R}_{i}\right)$ can be written as a linear superposition of the basis functions $\chi_{l}$

$$
\varphi\left(\mathbf{r}-\mathbf{R}_{i}\right)=\sum_{l} c_{i l} \chi_{l}\left(\mathbf{r}-\mathbf{R}_{i}\right)
$$

where $\left\{\chi_{l}\left(\mathbf{r}-\mathbf{R}_{i}\right)\right\}$ are usually the Gaussian basis functions, and the coefficients $c_{i l}$ can be obtained from the variational method.
Then the electronic density of the molecule is

$$
\rho(\mathbf{r})=|\psi(\mathbf{r})|^{2}=\sum_{i j k l} d_{i k, j l} \chi_{k}\left(\mathbf{r}-\mathbf{R}_{i}\right) \chi_{l}\left(\mathbf{r}-\mathbf{R}_{j}\right),
$$

where $d_{i k, j l}=2 c_{i k} c_{j l}$, which can be divided into two parts, namely, the net $(i=j)$ and overlap $(i \neq j)$ populations. The latter cannot be ignored in the presence of strong intramolecular covalency. Therefore, separating $\rho(\mathbf{r})$ into its atomic constituents is to split the overlap population. However, the way to achieve that is not unique. For example, we can introduce a set of weights $w_{i j k l}$ due to different criteria such that

$$
\begin{gathered}
\widetilde{d}_{i k, i k}=d_{i k, i k}+\sum_{j \neq i, l} w_{i j k l} d_{i k, j l} \int \chi_{k}\left(\mathbf{r}-\mathbf{R}_{i}\right) \chi_{l}\left(\mathbf{r}-\mathbf{R}_{j}\right) d \mathbf{r}, \\
\widetilde{d}_{j l, j l}=d_{j l, j l}+\sum_{i \neq j, k}\left(1-w_{i j k l}\right) d_{i k, j l} \int \chi_{k}\left(\mathbf{r}-\mathbf{R}_{i}\right) \\
\times \chi_{l}\left(\mathbf{r}-\mathbf{R}_{j}\right) d \mathbf{r}, \\
\widetilde{d}_{i k, i l}=d_{i k, i l},
\end{gathered}
$$

then we can rewrite Eq. (8) as

$$
\rho(\mathbf{r}) \simeq \sum_{i} \rho_{i}(\mathbf{r})=\sum_{i k l} \widetilde{d}_{i k, j l} \chi_{k}\left(\mathbf{r}-\mathbf{R}_{i}\right) \chi_{l}\left(\mathbf{r}-\mathbf{R}_{i}\right)
$$

where $\rho_{i}(\mathbf{r})$ is the atomic density of atom $i$.

In our previous studies, the overlap electronic density is equally separated, i.e., $w_{i j k l}=1 / 2$ in Eq. (9), similar to the MPA. ${ }^{11,12,17,18,31}$ In Table I, we present the electronic multipole moments of $\mathrm{SnCl}_{6}^{2-}, \mathrm{ScF}_{6}^{3-}, \mathrm{SiO}_{4}^{4-}, \mathrm{SeO}_{4}^{2-}, \mathrm{SO}_{4}^{2-}$, $\mathrm{ClO}_{4}^{-}, \mathrm{CO}_{3}^{2-}$, and $\mathrm{NO}_{3}^{-}$calculated using the MPA, and compare them with the $a b$ initio values. We note that for these symmetrical molecules, the MPA preserves total charge and zero dipole moment. However, for a molecular ion such as $\mathrm{V}$-shaped $\mathrm{NO}_{2}^{-}$or linear $\mathrm{CN}^{-}$, the Mulliken population

TABLE I. Electronic multipole moments of molecule $\left(A B_{n}\right)$ calculated from the Mulliken population analysis. The $a b$ initio values are shown in parentheses. All quantities are in atomic units.

\begin{tabular}{lcccc}
\hline \hline$A B_{n}{ }^{\mathrm{a}}$ & $\mu_{z}^{\mathrm{b}}$ & $\vartheta_{z z}$ & $\Omega_{z z z}$ & $\Phi_{z z z z}$ \\
\hline $\mathrm{CN}^{-}$ & $0.14(0.17)$ & $-27.35(-29.41)$ & $7.99(10.68)$ & $-143(-166)$ \\
$\mathrm{NO}_{2}^{-}$ & $0.57(0.26)$ & $-19.64(-21.64)$ & $-2.60(-5.87)$ & $-60(-76)$ \\
$\mathrm{NO}_{3}^{-}$ & $0(0)$ & $-15(-16)$ & $0(0)$ & $-38(-41)$ \\
$\mathrm{CO}_{3}^{2-}$ & $0(0)$ & $-17(-17)$ & $0(0)$ & $-48(-48)$ \\
$\mathrm{ClO}_{4}^{-}$ & $0(0)$ & $-111(-111)$ & $0(0)$ & $-548(-548)$ \\
$\mathrm{SO}_{4}^{2-}$ & $0(0)$ & $-119(-120)$ & $0(0)$ & $-620(-627)$ \\
$\mathrm{SeO}_{4}^{2-}$ & $0(0)$ & $-143(-143)$ & $0(0)$ & $-823(-816)$ \\
$\mathrm{SiO}_{4}^{4-}$ & $0(0)$ & $-157(-158)$ & $0(0)$ & $-1011(-1019)$ \\
$\mathrm{ScF}_{6}^{3-}$ & $0(0)$ & $-319(-318)$ & $0(0)$ & $-5112(-5071)$ \\
$\mathrm{SnCl}_{6}^{2-}$ & $0(0)$ & $-845(-844)$ & $0(0)$ & $-19834(-19668)$ \\
\hline \hline
\end{tabular}

${ }^{a}$ In the HF calculations, basis set D95* were used for $A B$ and $A B_{2}, 6-31 \mathrm{G}^{*}$ for $A B_{3}$ and $A B_{4}$, and $3-21 \mathrm{G}^{*}$ for $A B_{6}$.

${ }^{\mathrm{b}}$ The electrostatic moments $\mu$ (dipole), $\vartheta$ (quadrupole), $\Omega$ (octapole), and $\Phi$ (hexadecapole) refer to the center of mass of the molecule with the standard orientation defined in GAUSSIAN 98 (Ref. 30). 
seems inadequate. Given total charge and the dipole moment 0.26 a.u. of $\mathrm{NO}_{2}^{-}$obtained from the ab initio calculations (Table I), a population analysis which preserves these vales would give rise to $-0.092 e$ on $\mathrm{N}$ and $-0.454 e$ on $\mathrm{O}$. Whereas, using the MPA $\left(w_{i j k l}=1 / 2\right)$, the charges on the $\mathrm{N}$ and $\mathrm{O}$ atoms are $0.1624 e$ and $-0.5812 e$, respectively, which renders the dipole moment 0.57 atomic unit, overestimated by $120 \%$.

Therefore, it is desirable to determine $w_{i j k l}$ in such a way that the calculated multipole moments of the molecule are consistent with the ab initio values. One possible way is to evaluate $w_{i j k l}$ by fitting the ab initio charge density, as shown in Eqs. (9) and (10), with the values of multipole moments as constraints. An alternative way is to directly fit the charge density, Eq. (10), with $\widetilde{d}_{i k, j l}$ being the parameters and $\chi_{k}\left(\mathbf{r}-\mathbf{R}_{i}\right) \chi_{l}\left(\mathbf{r}-\mathbf{R}_{i}\right)$ being the dependent variables. To simplify the computation, only the radial parts of $\chi_{k}(\mathbf{r}$ $\left.-\mathbf{R}_{i}\right) \chi_{l}\left(\mathbf{r}-\mathbf{R}_{i}\right)$ were kept. This fitting population analysis (FPA) is similar to that proposed by Parker and his coworkers as an alternative implementation of the GK model. ${ }^{32}$

To obtain the atomic electron densities on the nitrogen and oxygen atoms by the above MPA or FPA of the electron density of the nitrite anion, GAUSSIAN 98 program package ${ }^{30}$ is employed to solve the Hartree-Fock-Roothan equations for $\mathrm{NO}_{2}^{-}$in the gas phase; the in-crystal ions will be discussed in Sec. V. The atomic-orbital basis sets used are a double-zeta basis with polarization functions (D95*) for the nitrogen and oxygen atoms. ${ }^{33}$ The optimized geometry of $\mathrm{NO}_{2}^{-}$with the $\mathrm{N}-\mathrm{O}$ bond length $R_{\mathrm{NO}}=1.233 \AA$ and the bond angle $\theta_{\mathrm{ONO}}$ $=116.6^{\circ}$ is comparable to the experimental geometry of $\mathrm{NO}_{2}^{-}$in the ferroelectric phase of $\mathrm{NaNO}_{2}$ with $R_{\mathrm{NO}}$ $=1.236 \AA$ and $\theta_{\mathrm{ONO}}=115.4^{\circ} .{ }^{34}$

The intramolecular interaction within the harmonicoscillator approximation can also be obtained from frequency analysis in GAUSSIAN $98 .{ }^{30}$ The lowest vibration frequency $(1192 \mathrm{~K})$ of $\mathrm{NO}_{2}^{-}$is considerably higher than the highest libration frequency $(318 \mathrm{~K})$ obtained from Raman spectroscopy ${ }^{35}$ as well as the order-disorder transition temperature $(437 \mathrm{~K})$. Therefore, it is justified to treat the internal motion of the nuclei in the $\mathrm{NO}_{2}^{-}$group within the harmonic approximation, or even as a rigid rotor.

Note that the polarizability of $\mathrm{NO}_{2}^{-}$at its optimized geometry is highly anisotropic, that is, with $\alpha_{x x}=7.820, \alpha_{y y}$ $=10.823$, and $\alpha_{z z}=23.825$ in atomic units (see Fig. 1 for coordinate convention). Thus, one would expect this polarization to seriously affect the intermolecular pair potentials, and thus render the rigid-ion approximation in question. We shall examine this question in the following section by comparing the intermolecular potentials obtained from this approach with those from ab initio HF calculations.

\section{INTERMOLECULAR POTENTIALS}

The ab initio HF calculations are performed by using GAUSSIAN 98 program package ${ }^{30}$ to scan the potential-energy surface of $\mathrm{NO}_{2}^{-}: \mathrm{Na}^{+}$and $\mathrm{NO}_{2}^{-}: \mathrm{NO}_{2}^{-}$dimers. The $\mathrm{D} 95^{*}$ basis set is used for the nitrogen and oxygen atoms. As for the sodium atoms, we used both the standard $6-31 G^{*}$ basis and the Slater-type orbitals for $\mathrm{Na}^{+}$taken from the Clementi and Roetti table, ${ }^{36}$ it turned out that the difference between them is small. In these calculations, the geometrical variables of $\mathrm{NO}_{2}^{-}$are frozen at their equilibrium values, since we showed previously that the $\mathrm{NO}_{2}^{-}$group in $\mathrm{NaNO}_{2}$ could be treated as a rigid rotor. However, in the HF calculations, the electronic structure is allowed to vary in order to minimize the total energy, thus the electronic polarization effects are included.

In our calculations of intermolecular interactions, one $\mathrm{NO}_{2}^{-}$is fixed with its center of mass being the origin of the coordinate system, the dipole vector pointing to the $y$ axis and the $\mathrm{O}-\mathrm{O}$ line being aligned parallel to the $z$ axis (see Fig. 1). Then, Cartesian coordinates $(x, y, z)$ are the position of $\mathrm{Na}^{+}$or the center of mass of another $\mathrm{NO}_{2}^{-}$.

In order to study the effects of different population analysis schemes on the pair potentials, we performed three different calculations based on our rigid-ion models: (i) MPA with pair potentials [Eq. (5)], (ii) FPA with pair potentials, and (iii) FPA with nonpair potentials [Eq. (4)], referred to as models I, II, and III, respectively. We shall show in the following that the electronic polarization effect could be revealed from examining the differences between models I and II, while errors due to the pairwise additive approximation could be analyzed from the differences between models II and III.

In Figs. 2 and 3, we compare the intermolecular potentials obtained from models I-III and those from the ab initio HF calculations. The results for the $\mathrm{NO}_{2}^{-}: \mathrm{Na}^{+}$dimer are shown in Fig. 2: we find in both models I and II that the overall shapes of the GK potentials as a function of molecular separation agree with the $a b$ initio results, with the lowest $\mathrm{NO}_{2}^{-}-\mathrm{Na}^{+}$potential energy emerging in the $(0,-R, 0)$ configuration; whereas model III predicts incorrectly the lowest potential energy in $(R, 0,0)$. It thus appears that in model II the errors caused by the pairwise additive approximation are compensated by the errors due to FPA.

On the other hand, the electronic polarization effect also manifests itself in Fig. 2 based on the following two observations. First, notice that our rigid-ion models I and II fit best to the $a b$ initio results for the $(R, 0,0)$ configuration [Fig. 2(d)]. We attribute that to the anisotropic polarizability of $\mathrm{NO}_{2}^{-}\left(\alpha_{x x}<\alpha_{y y}<\alpha_{z z}\right)$, thus the electronic cloud of $\mathrm{NO}_{2}^{-}$is most unlikely to be polarized along the $x$ direction. Second, for $(0, R, 0)$ [Fig. 2(a)], the minimum potential energy in model II is closer to the $a b$ initio values than model I, whereas the result reverses for $(0,-R, 0)$ [Fig. 2(c)]. To understand this, we observe that for $(0, R, 0), \mathrm{Na}^{+}$is closer to $\mathrm{N}$ than $\mathrm{O}$, thus in the $a b$ initio calculations the electrons were attracted toward the $\mathrm{N}$ atom, leading to a smaller dipole moment. Therefore, the results yielded by model II, which produced smaller dipole moment than model I, tend to be closer to the HF results for $(0, R, 0)$. Obviously, the situation reverses for $(0,-R, 0)$.

Similarly, we show in Fig. 3 the $\mathrm{NO}_{2}^{-}-\mathrm{NO}_{2}^{-}$intermolecular potentials using models I-III and the HF method. The configurations are chosen as follows: the two $\mathrm{NO}_{2}^{-}$molecules are initially parallel at their experimental lowtemperature separation (1.82 $⿱ 2,2.83 \AA, 2.69 \AA)$ and then 
TABLE II. Experimental and theoretical structural parameters for the $\operatorname{Im} 2 m$ phase (III) of $\mathrm{NaNO}_{2}$. Lattice constants are given in $\AA$.

\begin{tabular}{lcccc}
\hline \hline \multicolumn{1}{c}{ Parameters } & Experiment $^{\mathrm{a}}$ & Model I & Model II & Model III \\
\hline$a$ & 3.5024 & 3.3889 & 3.5013 & 3.7353 \\
$b$ & 5.5209 & 5.4542 & 5.5485 & 5.4257 \\
$c$ & 5.3789 & 4.9254 & 4.8403 & 4.9669 \\
$y / b$ of $\mathrm{N}(2 a)$ & 0.0781 & 0.0498 & 0.0433 & 0.0586 \\
$y / b$ of $\mathrm{Na}(2 a)$ & 0.5437 & 0.5537 & 0.5492 & 0.5437 \\
$y / b$ of $\mathrm{O}(4 d)$ & -0.0443 & -0.0704 & -0.0740 & -0.0610 \\
z/c of $\mathrm{O}(4 d)$ & 0.1965 & 0.2111 & 0.2151 & 0.2098 \\
\hline \hline
\end{tabular}

${ }^{a}$ From x-ray-diffraction experiments at $30 \mathrm{~K}$, see Ref. 37.

one of them rotates around each of the $x, y, z$ axes through its center of mass, as shown in Figs. 3(a)-3(c). The results of models I-III agree reasonably well with the $a b$ initio calculations. On closer examination, in Fig. 3(c) in angle ranges $0^{\circ}-90^{\circ}$ and $270^{\circ}-360^{\circ}$, model I fits better to the $a b$ initio results than models II and III. This feature would be important when the rotation of $\mathrm{NO}_{2}^{-}$around the $c$ axis dominates its rotations around the $a$ and $b$ axes, as will be demonstrated in Sec. IV.

Summarizing this section, in spite of the presence of electronic polarization when two molecules are brought closer, the intermolecular potentials for the $\mathrm{NO}_{2}^{-}: \mathrm{Na}^{+}$and $\mathrm{NO}_{2}^{-}: \mathrm{NO}_{2}^{-}$dimers could be correctly reproduced by using models I and II.

\section{MOLECULAR DYNAMICS SIMULATIONS}

After the potential-energy surface for $\mathrm{NaNO}_{2}$ has been obtained, we are prepared to undertake MD simulations. Long-range Coulombic interaction in the crystal is represented by electrostatic interaction among point charges calculated from the population analysis, while each of the shortrange pair potentials are fitted by using an exponentialpolynomial function accurate within $0.1 \%{ }^{18}$ In the following description, the $x, y, z$ directions correspond to the crystallographic $a, b, c$ directions of $\mathrm{NaNO}_{2}$, respectively, see Fig. 1.

\section{A. Lattice relaxation}

Before we proceed with the molecular dynamics simulations, we perform lattice relaxation for the ferroelectric structure of $\mathrm{NaNO}_{2}$ both with and without the $\operatorname{Im} 2 m$ space-group symmetry constraints. This relaxation procedure provides the crystal structure with zero force on each atom, that is, an energy extremum; it also produces a test to the PES because the resultant structures have to agree reasonably with the experimental data for further simulations to be reliable. We perform both static and dynamic relaxations: the static one is an application of the Newton-Raphson algorithm and usually results in finding a local minimum of the energy, and the dynamic one is a simulated annealing calculation for overcoming that limitation. We start the static lattice relaxation with the experimental parameters. In Table II we present the lattice and basis parameters deduced from the experiments and static relaxation. In all cases, the static relaxation pro- duced essentially the same structure that strongly resembles the experimental structure. Most of the lattice constants in the relaxed structure are shorter than the experimental values (by $3.7 \%, 1.5 \%$, and $8.5 \%$ for $a, b$, and $c$, respectively, in model I, and by $0.5 \%,-2.4 \%$, and $10 \%$ for $a, b$, and $c$, respectively, in model II). Hence the calculated volume is smaller than the experimental one by $13 \%$ for model I and $10 \%$ for model II, a common feature for simulations using the GK model, which will be addressed in more detail in the following section.

Next, we go on to relax the statically relaxed crystal structure to zero temperature using a simulated annealing algorithm, in which the amount of kinetic energy in the molecules slowly decreases over the course of the simulation. We find that the (zero-temperature) ground states in models I and II are close to the statically relaxed structures, whereas there are substantial changes taking place in model III. By monitoring the orientations of the nitrite ions, we find that the ground structure in model III, still orthorhombic with $a$ $=3.90494 \AA, b=4.8441 \AA$, and $c=5.0770 \AA$, is ferroelectric with the dipole moments of $\mathrm{NO}_{2}^{-}$aligned along the $a$ axis rather than the experimental $b$ axis. So we conclude that the PES given by models III did not reflect reality. This concurs with the previous discussion on the intermolecular potentials (Sec. III). In the following, we use only models I and II to simulate the phase transition in $\mathrm{NaNO}_{2}$.

\section{B. MD simulations}

Using the isoenthalpic, isobaric ensemble, our MD simulation is started with a zero-temperature zero-pressure orthorhombic cell $(4 a \times 4 b \times 4 c)$ consisting of 512 atoms. Periodic boundary conditions are imposed to simulate an infinite crystal. The MD calculations are carried out in the Parrinello-Rahman scheme ${ }^{38}$ which allows both the volume and the shape of the MD cell to vary with time. The calculation of the energies and forces was handled by the Ewald method. A time step of 0.002 ps was used to integrate the equations of motion. In our heating runs, we raise the temperature of the sample in stages, $20 \mathrm{~K}$ each time, up to 1000 $\mathrm{K}$. At each stage, the first 2000 time steps were employed to equilibrate the system, then 10,000 time steps were collected for subsequent statistical analysis. Since our simulations employ periodic boundary conditions, we cannot distinguish the incommensurate structure (i.e., phase II of solid $\mathrm{NaNO}_{2}$ ). 


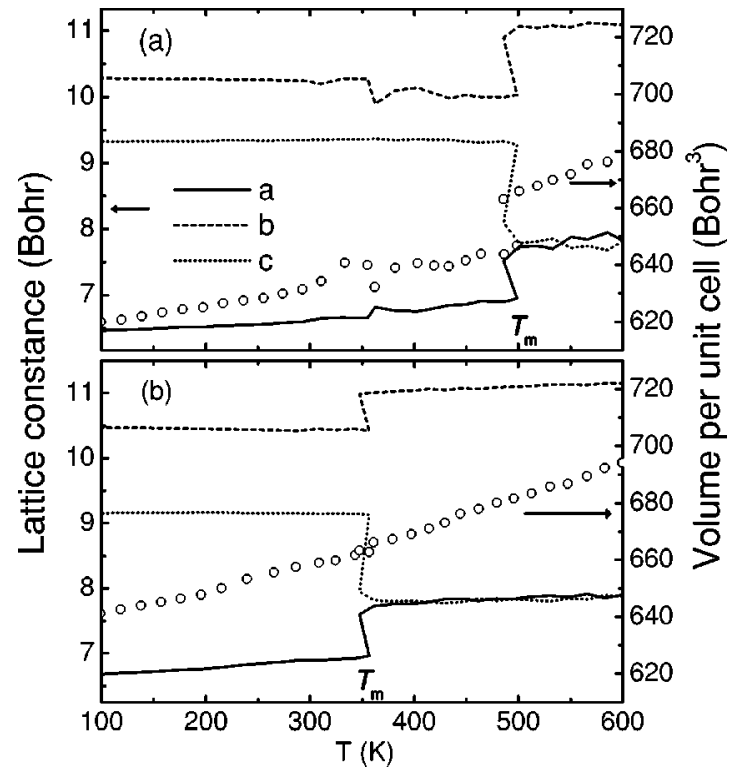

FIG. 4. Temperature variation of lattice constants $a, b, c$ (solid, dashed, and dotted lines, respectively; left scale) and volume of the unit cell (open circles; right scale) for (a) model I and (b) model II.

In Figs. 4-7, we demonstrate that as the MD cell is heated, it undergoes two phase transitions. In the first one, the system retains its orthorhombic structure with a change of space group from $\operatorname{Im} 2 \mathrm{~m}$ to $I \mathrm{mmm}$, in agreement with the experiments. The critical temperature $T_{\mathrm{C}}$ is around $550 \mathrm{~K}$ for model I and $550 \mathrm{~K}$ for model II. In the second transition, the crystal structure violently changes from orthorhombic to tetragonal at temperature $T_{\mathrm{m}}$ which is around $550 \mathrm{~K}$ for model $\mathrm{I}$ and $550 \mathrm{~K}$ for model II, as shown in Fig. 4. However, we argue that the crystal has already melted before this type of transition could be observed in reality.

To investigate the mechanism of the polarization reversal of $\mathrm{NO}_{2}^{-}$, we monitor the crystal polarization and display the results in Fig. 5. Let the dipole moment of anion $i$ be $\mathbf{m}_{i}$ and the quadrupole moment be $\boldsymbol{\vartheta}_{i}$ calculated by using the point charges on the $\mathrm{N}$ and $\mathrm{O}$ atoms. Then the mean dipole moment per anion at temperature $T$ is $\mathbf{M}(T)=\Sigma_{i}\left\langle\mathbf{m}_{i}\right\rangle / N$ where $N=128$ is the number of $\mathrm{NO}_{2}^{-}$in the MD cell and the brackets denote an average over the MD run. In addition, we define the antiferroelectric polarization as $\mathbf{Q}=\Sigma_{i} \exp \left(-\boldsymbol{\pi} \cdot \mathbf{R}_{i}\right)\left\langle\mathbf{m}_{i}\right\rangle / N$ where $\boldsymbol{\pi}=(\pi, \pi, \pi)$ and $\mathbf{R}_{i}$ is the lattice vectors associated with the $i$ th ion. Within our statistical uncertainty we find over all temperature range $M_{x}=M_{z}=\mathbf{Q}=0$, while $M_{y}\left(T<T_{\mathrm{C}}\right)>0$ and $M_{y}\left(T>T_{\mathrm{C}}\right)$ $=0$. This fact confirms that the transition taking place at $T_{\mathrm{C}}$ is the ferroelectric-paraelectric phase transition. Furthermore, we calculated the mean quadrupole moment $\boldsymbol{\Theta}=\Sigma_{i}\left\langle\boldsymbol{\vartheta}_{i}\right\rangle / N$. When the dipole vector of a $\mathrm{NO}_{2}^{-}$is aligned along the $b$ axis, $\vartheta_{x x} \simeq 0.00, \vartheta_{y y} \simeq-0.04$, and $\vartheta_{z z} \simeq-4.49$ for model I and $\vartheta_{x x} \simeq 0.00, \vartheta_{y y} \simeq-0.20$, and $\vartheta_{z z} \simeq-3.52$ for model II; thus $\left(\Theta_{x x}+\Theta_{y y}\right) / 2 \Theta_{z z} \ll 1$. This relation holds as the $\mathrm{NO}_{2}^{-}$ion rotates around the $c$ axis; nevertheless, one would expect $\left(\Theta_{x x}+\Theta_{y y}\right) / 2 \Theta_{z z}=1$ when the $\mathrm{NO}_{2}^{-}$ion rotates without directional preference. The fact that $\left(\Theta_{x x}+\Theta_{y y}\right) / 2 \Theta_{z z} \ll 1$ for $T<T_{\mathrm{m}}$ (Fig. 5) reveals that the $\mathrm{NO}_{2}^{-}$anions rotate primarily
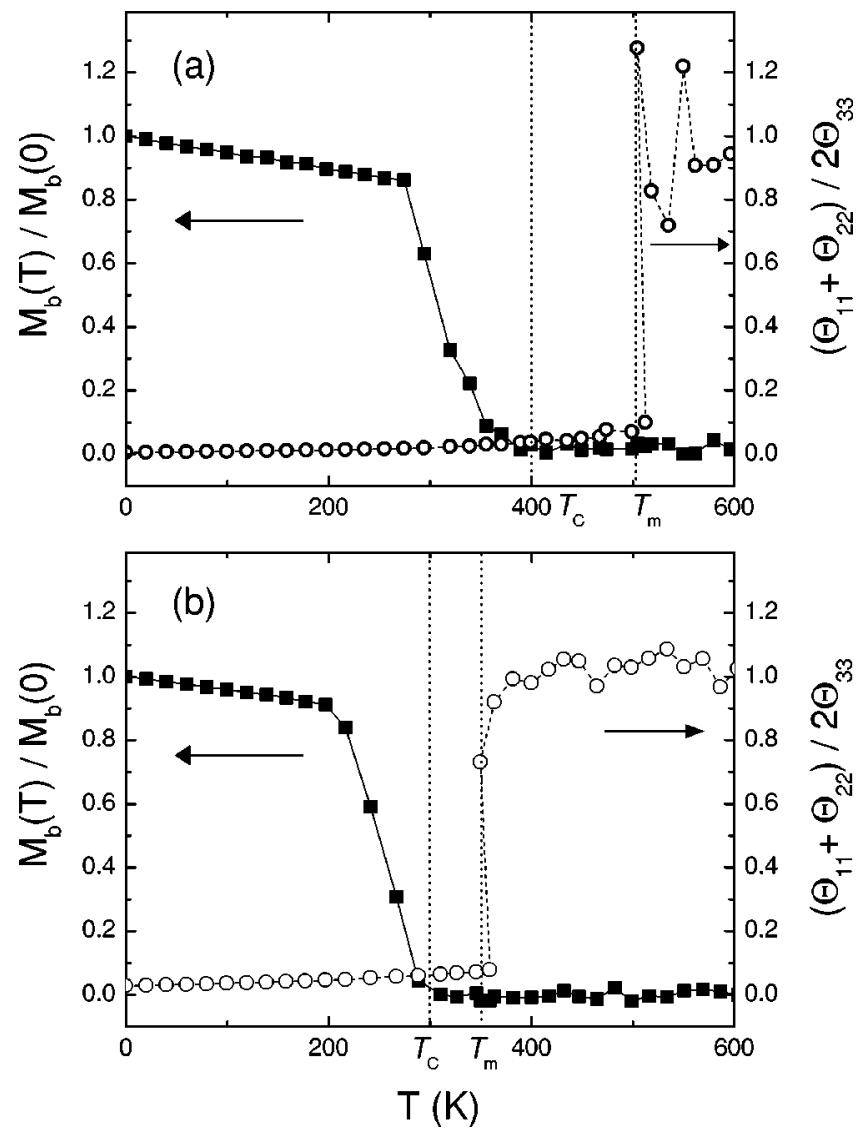

FIG. 5. Mean dipole moment $M_{b}(T)$ and quadrupole moment $\Theta$ of the whole $\mathrm{NaNO}_{2}$ crystal as a function of temperature for the MD runs for (a) model I and (b) model II.

about the $c$ axis. When $T>T_{\mathrm{m}},\left(\Theta_{x x}+\Theta_{y y}\right) / 2 \Theta_{z z} \simeq 1$, i.e., $\mathrm{NaNO}_{2}$ becomes an orientational liquid.

Further, in Fig. 6 we show the mean-square atomic displacements $U_{i i}=\left\langle u_{i}^{2}\right\rangle$ where $i=1,2,3$ denotes the displacements along the $a, b, c$ axes, respectively. Different models of $\mathrm{NO}_{2}^{-}$reversal are expected to satisfy the following conditions. (1) Rotation around the $c$ axis: $U_{22}(\mathrm{~N}), U_{33}(\mathrm{~N})$ $<U_{11}(\mathrm{~N})$ and $U_{22}(\mathrm{O}), U_{33}(\mathrm{O})<U_{11}(\mathrm{O})$. (2) Rotation around the $a$ axis: $U_{11}(\mathrm{~N}), U_{22}(\mathrm{~N})<U_{33}(\mathrm{~N})$ and $U_{11}(\mathrm{O}), U_{33}(\mathrm{O})<U_{22}(\mathrm{O})$. This figure relates to recent $\mathrm{x}$-ray experiments which used the same quantities to investigate the polarization reversal mechanism. ${ }^{4,5}$ The experiments confirmed that the first condition holds for both ferroelectric and paraelectric phases. Another important feature revealed by the experiments is that $U_{22}(\mathrm{Na}), U_{33}(\mathrm{Na})<U_{11}(\mathrm{Na})$ in the ferroelectric phase, whereas $U_{11}(\mathrm{Na}), U_{33}(\mathrm{Na})<U_{22}(\mathrm{Na})$ in the paraelectric phase. That is, $U_{11}(\mathrm{Na})$ and $U_{22}(\mathrm{Na})$ are reversed across $T_{\mathrm{C}}$. These features are reproduced in Fig. 6 with exception of $U_{11}(\mathrm{O}), U_{33}(\mathrm{O})<U_{22}(\mathrm{O})$ in the paraelectric phase. This means the $\mathrm{NO}_{2}^{-}$motions in our simulations are more mobile than those in the real crystal, rendering the simulated transition temperatures lower than the experimental values of $T_{\mathrm{C}} \simeq 437 \mathrm{~K}$ and the melting temperature $550 \mathrm{~K}$. In other words, the barriers to $\mathrm{NO}_{2}^{-}$rotation in our models are too small. 

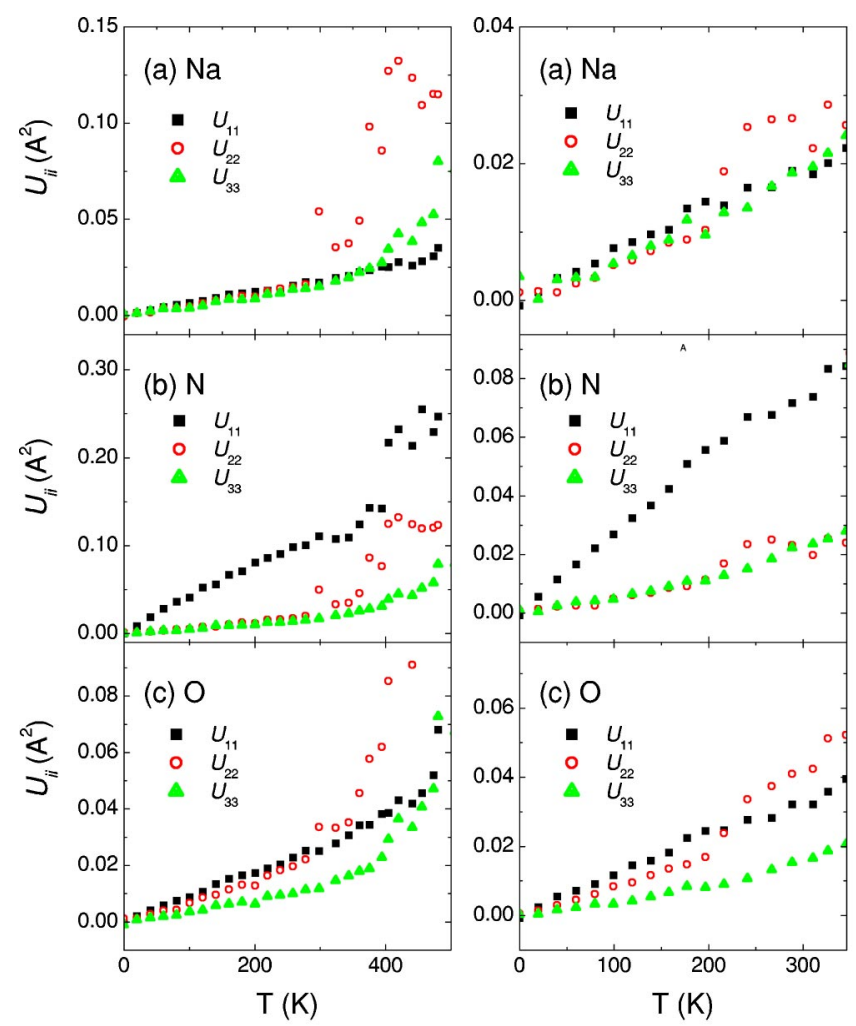

FIG. 6. Diagonal elements of the mean-square atomic displacements $U_{i i}$ vs temperature. (a) $\mathrm{Na}$, (b) $\mathrm{N}$, and (c) $\mathrm{O}$ atom.

In addition, in Fig. 7, we show the average crystal structures of $\mathrm{NaNO}_{2}$ at different temperatures. The ellipsoids in these pictures represent the root-mean-square deviations of the atoms from their average positions and thus indicate the thermal motions of these atoms. The $c$-axis rotation mode can be clearly seen, particularly in Fig. 7(c).

It is worth mentioning the less desirable agreement between theoretical and experimental volumes, namely, the $13 \%$ discrepancy for model I and $10 \%$ for model II. To address this we make one simple change: by following Waldman and Gordon, ${ }^{39}$ we increase the kinetic-energy term in the Gordon-Kim potentials by $5 \%$, this reduces the discrepancy to $9 \%$ for model I and $6 \%$ for model II. Having done this we rerun the MD to obtain values of $T_{c}$ of $360 \mathrm{~K}$ for model I and $303 \mathrm{~K}$ for model II. While this change worsens the value for model I, the value for model II is virtually unchanged. And in both cases the transition mechanism is unaltered. Thus the slight hardening of the short-range potentials removes most of the volume discrepancies. However, there is no material change in the mechanism of the phase transition. This robustness of the results with respect to minor variations in the potential demonstrates that our basic conclusions remain valid.

\section{Rotational barriers}

Based on the above simulation results, the order-disorder phase transition in $\mathrm{NaNO}_{2}$ involves the rotation of the nitrite ions. We devise a scheme to calculate the rotational barriers: Starting from the experimental ferroelectric structure ${ }^{34}$ taken

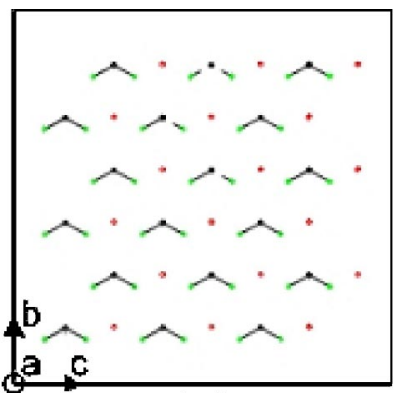

(a)

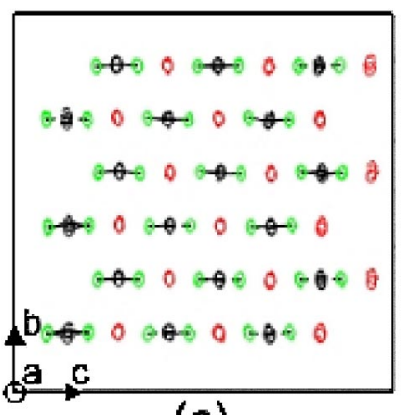

(c)

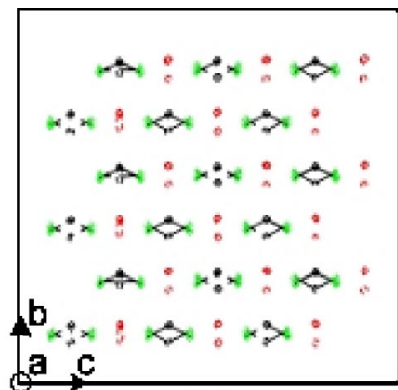

(b)

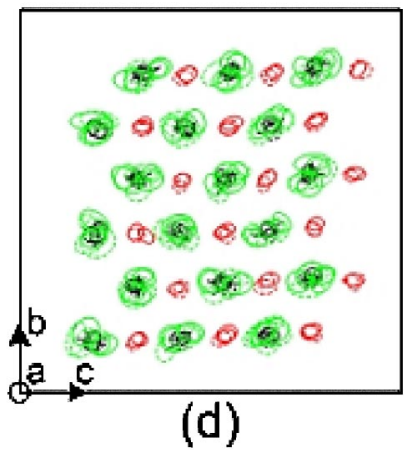

FIG. 7. Atomic positions of $\mathrm{NaNO}_{2}$ viewed from the $a$ direction obtained from the MD simulation for model I at (a) $T=198 \mathrm{~K}$, (b) $T=320 \mathrm{~K}$, (c) $T=449 \mathrm{~K}$, and (d) $T=535 \mathrm{~K}$.

to be the zero-energy state, we rotate one of the two nitrite ions in the unit cell around the $a, b$, and $c$ axes with the center of mass of the nitrite ion being fixed. The results are shown in Fig. 8. The bottom of each barrier is located at zero rotational angle that denotes the ferroelectric structure. For both models I and II, the rotation around the $c$ axis has an energy barrier 5-10 times smaller than those of the other rotations, which is a characteristic of nitrites. ${ }^{31}$ Hence, our calculations unambiguously reveal that the reorientation of $\mathrm{NO}_{2}^{-}$in the paraelectric phase occur essentially by rotations around the $c$ axis. Moreover, the barriers calculated in model I are higher than those in model II, confirming that the transition temperatures predicted by model I are higher than those predicted by model II.
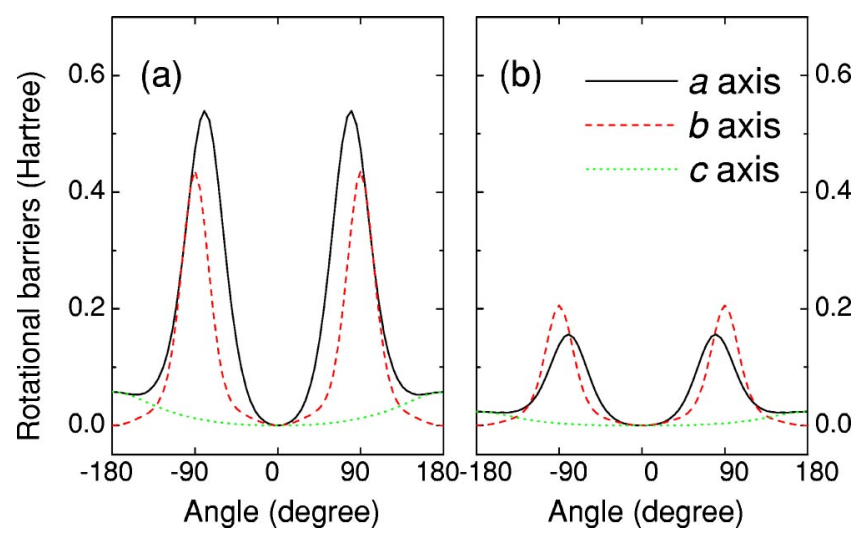

FIG. 8. Rotational barriers of one of the two nitrite ions in the unit cell around the $a, b$, and $c$ axes with its center of mass fixed. (a) Model I and (b) Model II. 


\section{CRYSTAL-FIELD EFFECTS}

Generally speaking, our simulations predict lower transition temperatures than the experimental values. We believe that the main reason is due to underestimation of anion polarization effect in solid $\mathrm{NaNO}_{2}$. In model II, $\mathrm{NO}_{2}^{-}$possesses the same dipole moment as in its gas phase, thus the polarization effect is totally neglected. In model I, the dipole moment has been enhanced by Mulliken charges-such enhancement could be interpreted as taking into account the polarization effect in the mean-field sense ${ }^{40}$ - thus leading to higher transition temperatures than those obtained from model II. The above comparison indicates that more improvement for dealing with the anion polarization effect would be needed to raise the calculated transition temperatures closer to the experimental values.

To manifest the substantial crystal-field effects on the $\mathrm{NO}_{2}^{-}$ions, we perform the following $\mathrm{HF}$ calculations: the crystal field of ferroelectric $\mathrm{NaNO}_{2}$ is simulated by placing the nitrite ion and its six nearest $\mathrm{Na}^{+}$cations at the center of a $4 \times 4 \times 4$ orthorhombic point-charge lattice with spacings equal to the experimental lattice parameters. Charges in the faces of the lattice are scaled to maintain overall neutrality. All anions except the central $\mathrm{NO}_{2}^{-}$are represented by single point charges on their centers of mass. Hence, there are 168 point charges surrounding the $\mathrm{NO}_{2}^{-}\left(\mathrm{Na}^{+}\right)_{6}$ cluster. Calculations of this type were proposed by Fowler and co-workers in the studies of monatomic ions ${ }^{23}$ and cyanides. ${ }^{41}$ The same basis set $\mathrm{D} 95^{*}$ is employed for the in-crystal $\mathrm{NO}_{2}^{-}$ion as for the free $\mathrm{NO}_{2}^{-}$ion, while the minimal basis set STO-3G is used for the $\mathrm{Na}^{+}$ions. The cations, however, are relatively insensitive to the crystal environment and they are included here only to account for their compressing effect on the $\mathrm{NO}_{2}^{-}$ wave functions. We find that adding extra basis functions to $\mathrm{Na}^{+}$will not change the results significantly. The central $\mathrm{NO}_{2}^{-}$initially points in the $b$ direction as in the ferroelectric phase of $\mathrm{NaNO}_{2}$. Subsequently, we rotate the $\mathrm{NO}_{2}^{-}$about the $a, b$, and $c$ axes through its center of mass.

As shown in Fig. 9(a), the dipole moment of the central $\mathrm{NO}_{2}^{-}$changes considerably as it rotates, indicating strong crystal-field effects on the reorientation of $\mathrm{NO}_{2}^{-}$. We also find that the dipole moment is sensitive to the location of the rotation center of $\mathrm{NO}_{2}^{-}$. In the context of population analysis, increase of the dipole moment of $\mathrm{NO}_{2}^{-}$implies that more electrons are distributed on the $\mathrm{O}$ atom, i.e., electrons are flowing from the nitrogen atom to the oxygen atoms. Conversely, decrease of the dipole moment indicates a reversal in electron transfer. Therefore, we have demonstrated considerable intramolecular charge transfer, although the intermolecular charge transfer is usually small in ionic crystals.

Although strong crystal-field effects have been revealed by these $a b$ initio calculations, the rotational barriers obtained from the polarizable-ion model [Fig. 9(b)] are in qualitative agreement with those from the rigid-ion models [Figs. 8(a) and 8(b)], confirming that the rigid-ion model is capable of describing the phase behavior in $\mathrm{NaNO}_{2}$.
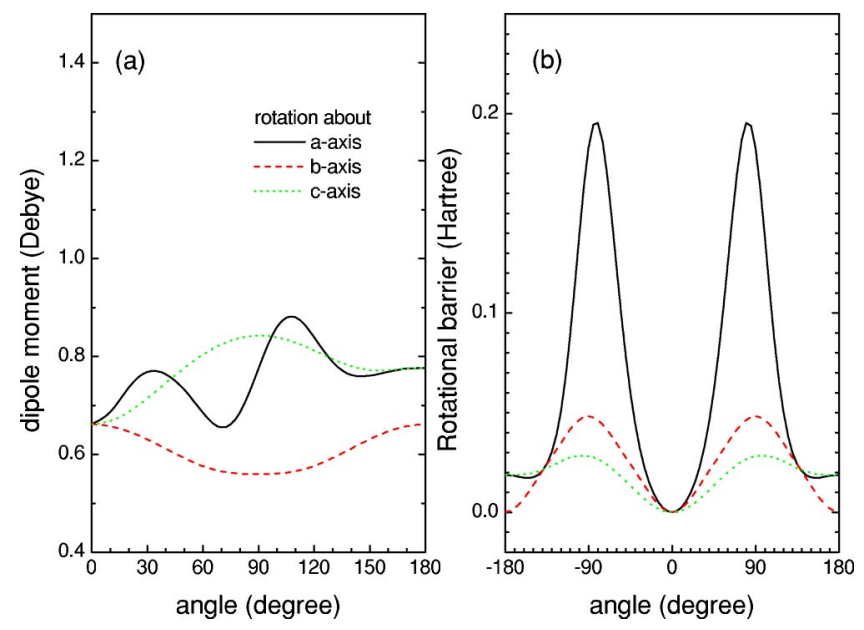

FIG. 9. HF calculations on a $\mathrm{NO}_{2}^{-}\left(\mathrm{Na}^{+}\right)_{6}$ cluster embedded in a lattice of point charges with $\mathrm{NO}_{2}^{-}$rotating around the $a, b, c$ axes through its center of mass. (a) Dipole moment of $\mathrm{NO}_{2}^{-}$and (b) rotational barriers.

\section{CONCLUDING REMARKS}

We have presented MD simulations of $\mathrm{NaNO}_{2}$ using a hybrid a priori method consisting of $a b$ initio calculations and Gordon-Kim electron-gas theory to analytically calculate the crystal potential surface. This method has been carefully examined by using different population analysis methods. We have carried out $a b$ initio Hartree-Fock calculations of the intermolecular interactions for $\mathrm{NO}_{2}^{-}: \mathrm{Na}^{+}$and $\mathrm{NO}_{2}^{-}: \mathrm{NO}_{2}^{-}$dimers and concluded that the pair potentials of the rigid-ion model can correctly reproduce the $a b$ initio results. We demonstrated that a rigid-ion model is capable of describing phase behavior in solid $\mathrm{NaNO}_{2}$.

We also addressed the crystal-field effects on the $\mathrm{NO}_{2}^{-}$ion by performing Hartree-Fock calculations on a $\mathrm{NO}_{2}^{-}\left(\mathrm{Na}^{+}\right)_{6}$ cluster embedded in a lattice of point charges. We conclude that the partial charges on the nitrogen and oxygen atoms are fluctuating in solid $\mathrm{NaNO}_{2}$ in response to changing crystalfield environments, which arise particularly from the rotation of the nitrite ions. Our MD simulations are based on two rigid-ion models using MPA and FPA, respectively. The model using MPA, which enhances the dipole moment of $\mathrm{NO}_{2}^{-}$in the gas phase, gives rise to more comparable results with the experiments. Such enhancement stabilizes the ferroelectric structure by raising the rotational barriers of $\mathrm{NO}_{2}^{-}$, thus mimicing the anion polarization effect in the mean-field sense. To quantitatively simulate $\mathrm{NaNO}_{2}$, a more elaborate polarizable-ion model is needed.

\section{ACKNOWLEDGMENTS}

Helpful discussions with Dr. L. L. Boyer are gratefully acknowledged. This work was supported by the Nebraska Research Initiative, the Nebraska EPSCoR-NSF Grant No. EPS-9720643, and Department of the Army Grants Nos. DAAG 55-98-1-0273 and DAAG 55-99-1-0106. W.N.M. is grateful for the support from the Office of Naval Research. 
*Electronic address: wgyin@yahoo.com

${ }^{1}$ S. Sawada, S. Nomura, S. Fujii, and I. Yoshida, Phys. Rev. Lett. 1, 320 (1958).

${ }^{2}$ M. E. Lines and A. M. Glass, Principles and Applications of Ferroelectrics and Related Materials (Clarendon, Oxford, 1977).

${ }^{3}$ A.V. Fokin, Y.A. Kumzerov, N.M. Okuneva, A.A. Naberezhnov, S.B. Vakhrushev, I.V. Golosovsky, and A.I. Kurbakov, Phys. Rev. Lett. 89, 175503 (2002).

${ }^{4}$ T. Gohda, M. Ichikawa, T. Gustafsson, and I. Olovsson, Phys. Rev. B 63, 014101 (2000).

${ }^{5}$ K. Komatsu, K. Itoh, and E. Nakamura, J. Phys. Soc. Jpn. 57, 2836 (1988).

${ }^{6}$ K.D. Ehrhardt and K.H. Michel, Phys. Rev. Lett. 46, 291 (1981).

${ }^{7}$ W. Kinase and K. Takahashi, J. Phys. Soc. Jpn. 61, 329 (1992).

${ }^{8}$ M.L. Klein, I.R. McDonald, and Y. Ozaki, Phys. Rev. Lett. 48, 1197 (1982).

${ }^{9}$ M.L. Klein and I.R. McDonald, Proc. R. Soc. London, Ser. A A382, 471 (1982).

${ }^{10}$ R.M. Lynden-bell, R.W. Impey, and M.L. Klein, Chem. Phys. 109, 25 (1986).

${ }^{11}$ H.M. Lu and J.R. Hardy, Solid State Commun. 87, 1151 (1993).

${ }^{12}$ H.M. Lu and J.R. Hardy, Phys. Rev. Lett. 64, 661 (1990).

${ }^{13}$ P.J. Edwardson, L.L. Boyer, R.L. Newman, D.H. Fox, J.R. Hardy, J.W. Flocken, R.A. Guenther, and W. Mei, Phys. Rev. B 39, 9738 (1989).

${ }^{14}$ H.M. Lu and J.R. Hardy, Phys. Rev. B 42, 8339 (1990).

${ }^{15}$ J. Liu, C. Duan, W.N. Mei, R.W. Smith, and J.R. Hardy, J. Solid State Chem. 163, 294 (2002).

${ }^{16}$ D. Liu, H.M. Lu, F.G. Ullman, and J.R. Hardy, Phys. Rev. B 43, 6202 (1991).

${ }^{17}$ J. Liu, C. Duan, W.N. Mei, R.W. Smith, and J.R. Hardy, J. Chem. Phys. 116, 3864 (2002).

${ }^{18}$ H.M. Lu and J.R. Hardy, Phys. Rev. B 44, 7215 (1991).

${ }^{19}$ J. Liu, C. Duan, M.M. Ossowski, W.N. Mei, R.W. Smith, and J.R.
Hardy, J. Solid State Chem. 160, 222 (2001).

${ }^{20}$ J. Liu, C. Duan, M.M. Ossowski, W.N. Mei, R.W. Smith, and J.R. Hardy, J. Phys. Chem. Solids 63, 409 (2002).

${ }^{21}$ W. Hehre, L. Radom, P. Schleyer, and J. Pople, Ab Initio Molecular Orbital Theory (Wiley, New York, 1986), p. 336.

${ }^{22}$ P. Ravindran, A. Delin, B. Johansson, O. Eriksson, and J.M. Wills, Phys. Rev. B 59, 1776 (1999).

${ }^{23}$ P.W. Fowler and P.A. Madden, Phys. Rev. B 29, 1035 (1984).

${ }^{24}$ Y.S. Kim and R.G. Gordon, Phys. Rev. B 9, 3548 (1974).

${ }^{25}$ A.J. Cohen and R.G. Gordon, Phys. Rev. B 12, 3228 (1975).

${ }^{26}$ L.L. Boyer, Phys. Rev. Lett. 42, 584 (1979).

${ }^{27}$ M. Sepliarsky, S.R. Phillpot, D. Wolf, M.G. Stachiotti, and R.L. Migoni, Appl. Phys. Lett. 76, 3986 (2000).

${ }^{28}$ R.G. Gordon and Y.S. Kim, J. Chem. Phys. 56, 3122 (1972).

${ }^{29}$ M. Waldman and R.G. Gordon, J. Chem. Phys. 71, 1340 (1979).

${ }^{30}$ M. J. Frisch et al., GAUSSIAN 98, Revision A.11.3 (Gaussian, Inc., Pittsburgh, PA, 2002).

${ }^{31}$ C.G. Duan, W.N. Mei, R.W. Smith, J. Liu, M.M. Ossowski, and J.R. Hardy, Phys. Rev. B 63, 144105 (2001).

${ }^{32}$ G.A. Parker, R.L. Snow, and R.T. Pack, Chem. Phys. Lett. 33, 399 (1975).

${ }^{33}$ A. Banerjee, R. Shepard, and J. Simons, J. Chem. Phys. 73, 1814 (1980).

${ }^{34}$ M.I. Kay, Ferroelectrics 4, 235 (1972).

${ }^{35}$ C.M. Hartwig, E. Wiener-Avnear, and S.P.S. Porto, Phys. Rev. B 5, 79 (1972).

${ }^{36}$ E. Clementi and C. Roetti, At. Data Nucl. Data Tables 14, 177 (1974).

${ }^{37}$ T. Gohda, M. Ichikawa, T. Gustafsson, and I. Olovsson, Acta Crystallogr., Sect. B: Struct. Sci. B56, 11 (2000).

${ }^{38}$ M. Parrinello and A. Rahman, Phys. Rev. Lett. 45, 1196 (1980).

${ }^{39}$ M. Waldman and R.G. Gordon, J. Chem. Phys. 71, 1325 (1979).

${ }^{40}$ S. Rick, S. Stuart, and B. Berne, J. Chem. Phys. 101, 6141 (1994).

${ }^{41}$ P.W. Fowler and M.L. Klein, J. Chem. Phys. 85, 3913 (1986). 